\title{
Multimodal Chemical and Functional Imaging of Nanoscale Transformations in Ferroelectric Thin Films
}

Anton V. Ievlev ${ }^{1,2}$, Chance Brown ${ }^{1,2}$, Petro Maksymovych ${ }^{1,2}$, Sergei V. Kalinin ${ }^{1,2}$ and Olga S. Ovchinnikova ${ }^{1,2}$

${ }^{1}$ Center for Nanophase Materials Sciences, Oak Ridge National Laboratory, Oak Ridge, TN, USA

${ }^{2}$ Institute for Functional Imaging of Materials, Oak Ridge National Laboratory, Oak Ridge, TN, USA

Continuing miniaturization of portable electronic and mechanical devices requires development of the novel nanoscale elements. This can be realized using ferroelectric materials, which exhibit a unique set of functional properties, including pyroelectricity, piezoelectricity, non-linear optical activity and others. These properties enable a wide range of practical applications from sensors and actuators to data storage and processing devices [1-3]. However, functionality of ferroelectric materials is defined by the number of physical and chemical phenomena on the nanoscale, which requires using of the nanoscale imaging techniques allowing functional and chemical investigations. Atomic Force Microscopy (AFM) is one of such techniques. Various AFM electric modes are widely used for characterization of the ferroelectric physical properties on the nanoscale [4]. But at the same time, chemical contribution in the ferroelectric phenomena in the most cases are completely ignored due to the lack of tools for nanoscale chemical investigations. These studies can be carried out by combination of AFM with concomitant microscopic or spectroscopic technique, providing chemical or crystallographic information about studied sample [5]. One of such cases if offered by combination with Time-of-Flight Secondary Ion Mass Spectrometry (ToF-SIMS), which allows local investigation of the chemical composition on the sample surface and in the sample bulk [6].

Here, we utilize this approach for correlated investigations of the functional and chemical phenomena in $\mathrm{PbZr}_{0.2} \mathrm{Ti}_{0.8} \mathrm{O}_{3}$ (PZT) ferroelectric thin film. Studies were carried out using ION.TOF 5 NCS ToF-SIMS combined with Nanoscan AFM in the same vacuum chamber. $\mathrm{O}_{2}$ ion gun has been used for the cleaning of the PZT surface before measurements. In the studies, we used AFM to induce local ferroelectric switching by the electric field of the biased tip (Fig. 1) and study associated local changes in the chemistry of the film by ToF-SIMS. Investigations showed 3-5\% change in the spatial concentrations of the base chemical elements $(\mathrm{Pb}, \mathrm{Ti}, \mathrm{Zr}$ ) close to the sample surface (Fig. 2). All the changes were localized within 3.5-nm from the surface and formed double layer with higher concentrations on the top and lower concentrations at about $2.5 \mathrm{~nm}$ depth. This redistribution of ions is caused by the screening process and minimizes depolarization electric filed produced by the boundary charges surfaces. Observed phenomenon was also found to be reversible by application of the electric field of opposite polarity, which confirms its ferroelectric origin.

Results of the study shed light on the chemical phenomena associated with ferroelectric properties and are important for their fundamentals investigations and practical applications [7].

References:

[1] Scott J.F., Science 315 (2007), p. 954

[2] Sohler W. et.al, Opt. Photon. News 19 (2008), p. 24.

[3] Ievlev A.V. et.al, Nat. Phys 10 (2014), p. 59.

[4] Kalinin S.V.et.al, Rep. Prog Phys 73 (2010), p. 056502. 
[5] Ievlev, A.V. et.al, ACS Nano 9(12) (2015), p. 12442.

[6] Ievlev, A.V. et.al, ACS Appl. Mat. \& Interf. 8 (2016), p. 29588.

[7] Research supported by Oak Ridge National Laboratory's Center for Nanophase Materials Sciences (CNMS), which is a U.S. Department of Energy, Office of Science User Facility.
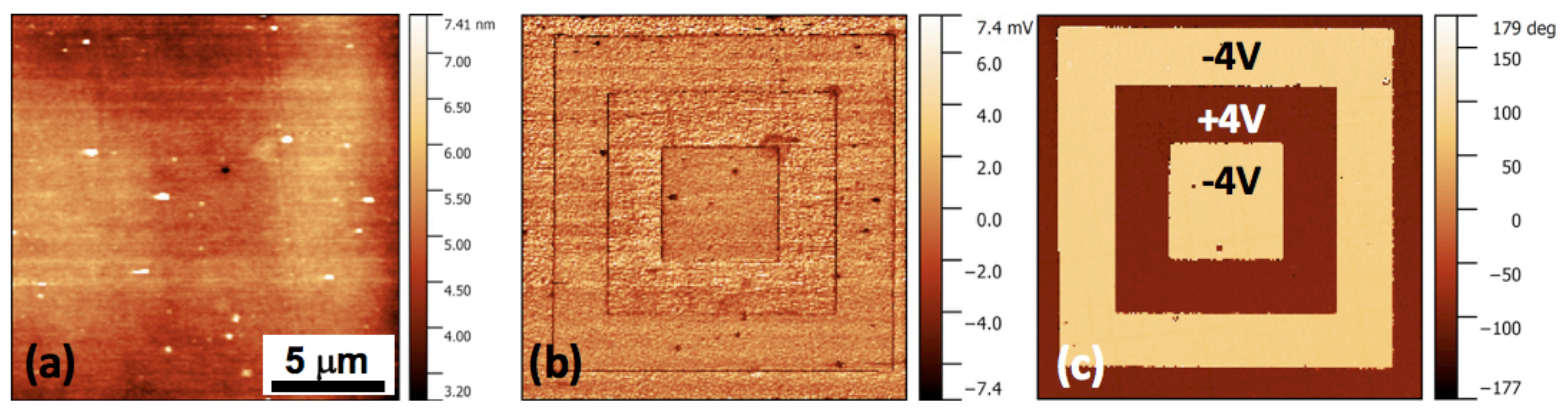

Figure 1. Results of the local polarization switching by the biased AFM tip. (a) AFM topography; (b), (c) ferroelectric domain structure measured by piezoresponse force microscopy (PFM) mode, signals of piezoresponse amplitude (b) and phase (c).

(a)
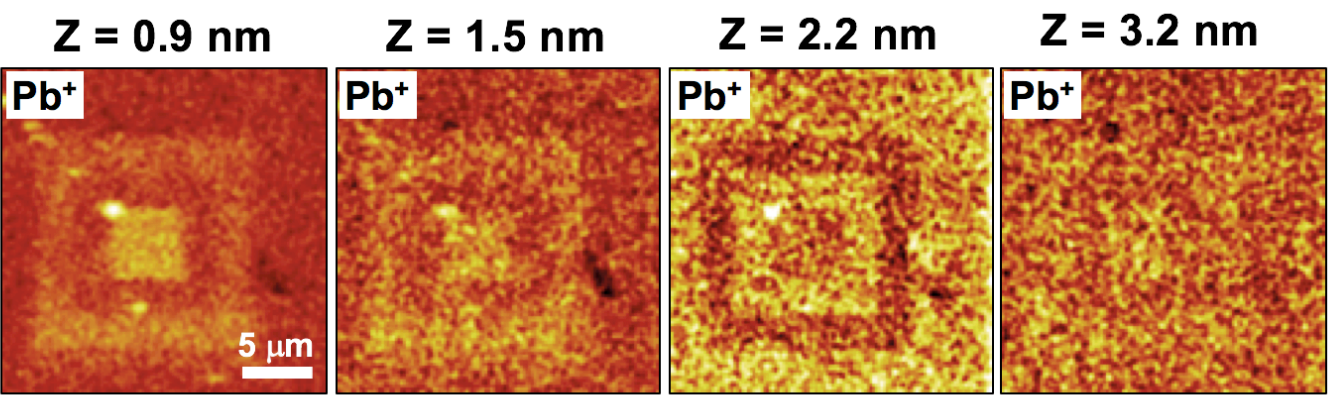

(b)
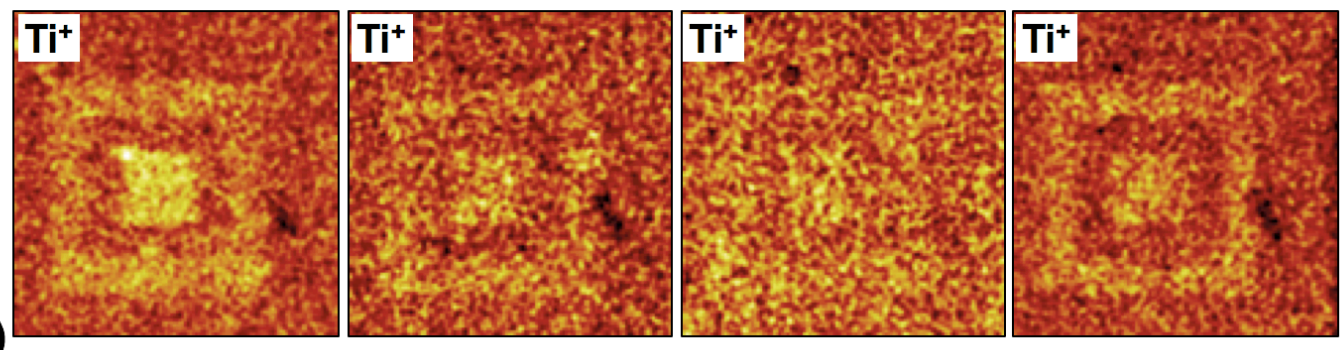

Figure 2. ToF-SIMS investigation of the ferroelectrically switched region. Local changes in concentration of $\mathrm{Pb}^{+}$(a) and $\mathrm{Ti}^{+}$(b) ions as a function of depth. 